\title{
Robust discrete-time nonlinear sliding mode controller with plant uncertainties
}

\author{
Nikhil Kumar Yadav ${ }^{1}$, R. K. Singh ${ }^{2 \#}$ \\ ${ }^{1 *}$ Department of Electrical Engineering, Motilal Nehru National Institute of Technology Allahabad (UP) - 211004, INDIA \\ ${ }^{\# 2}$ Department of Mechanical Engineering, Motilal Nehru National Institute of Technology Allahabad (UP) - 211004, INDIA \\ ${ }^{1 *}$ Corresponding Author: e-mail: nyadav24@gmail.com Mob: +91-9818486179, ${ }^{2 *}$ rksingh@mnnit.ac.in
}

\begin{abstract}
This paper addresses the new control algorithm, by designing the asymptotically stable nonlinear sliding surface with investigation of the states. This proposed algorithm leads to solve the problem of unstable systems, by proving the asymptotic stability of a class of uncertain discrete-time systems. A particular linear transformation is being defined to transform the discrete-time system and asymptotic stability is proved for designed nonlinear sliding surface, which leads to show stability of the system. The states of the plant's will be brought down on a proven asymptotic stable nonlinear sliding surface by the proposed control law and they will be remained on stable nonlinear sliding surface for all future times. This proposed technique will be more useful for enhancing the stability of the real world nonlinear systems. The application of proposed algorithm in magnetic tape drive-servo motors and avionic systems, where the parametric uncertainties are occurring frequently.
\end{abstract}

Keywords: Discrete-time system, linear matrix inequality (LMI), nonlinear system. nonlinear sliding surface, sliding mode control.

DOI: http://dx.doi.org/10.4314/ijest.v4i1.5S

\section{Introduction}

The implementation of discrete-time system by using computers, which will provide the more accurate response by using the microprocessor or microcontroller. The time delay is a source of instability for discrete-time systems. In many applications, time delays are introduced in the model of physical systems due to measurement, transmission and transport tags, computational delays of unmodeled inertia of system components. The time delays are quite common in all types of engineering systems such as chemical process control, delay in the actuator operation of mechanical systems and economic systems etc. Compared to the systems without delay, the presence of the delay makes the system more difficult to achieve its satisfactory performance of the systems. In recent years there is a great interest of the researcher for the processes having the state delays.

The author (Milosavljevic, 1985) was first researchers to formally propose that the sampling process in discrete-time systems can limit the existence of the sliding mode. Later on, (Sapturk et al., 1987) suggested that a reaching condition should widely be used in current DSMC systems. The technique (Bartoszewicz. et al., 1998) proposed a control algorithm that guarantees finite-time convergence of the state trajectory to the sliding surface. There in reference (Gao. et al., 1995) clarified the concept of discretetime sliding mode control by presenting several new sufficient conditions to illustrate the necessity of using the discrete-time sliding mode control.

The concept of sliding mode control (SMC) in recent years has drawn the attention of researchers. Sliding mode control is a robust control method which can handle both linear and nonlinear systems, hampered with parametric uncertainties and unexpected disturbances (Slotine et al., 1983, Utkin, 1972). It has been developed in the continuous time domain to a great extent. Sliding mode control is a methodology in which an appropriate input is provided so that the system states are confined to the desired sub-manifold of the state space. (Furuta, 1990 ) proposed discrete time sliding mode control laws where the control input includes state feedback terms in three different feedback coefficients. The discrete time sliding mode control has been mainly 
applied to stabilization problems. However in stabilization problems only bounded stability is guaranteed if the uncertainties in the system do not vanish at the equilibrium points. In paper ( Utkin, 1971) deals with the mismatch uncertainties for discrete adaptive sliding mode control for the nonlinear system for the output to track a sufficiently smooth desired trajectory. In paper of (Furuta, 1990) nonlinear sliding surface is created for varying the damping factor for improving the performance of the multi-input and multi-output linear systems with matched conditions. Some of the concepts and theoretical advances of continuous time sliding mode control are covered in literature (Won et al., 1995, Bandhyopadhyay et al., 2009) and the references there in. Due to flexibility of implementation, large classes of continuous systems are controlled by digital signal processors and microprocessors. To analyze the sampling time effect, discrete sliding mode control (SMC) is well studied in the literature (Hung et al., 1993, Ghezawi et al., 1983, Young et al., 1999, Edward et al., 1998, Gao. et al., 1995, Spurgeon, 1992, Golo et al., 2000, Furukawa et al., 1983, Hu et al., 2009, Janardhanan et al., 2006, Fiagbedzi et al., 1986, Jafarov et al., 2000, Utkin, 1977, Lee et al., 2001 and Lan et al., 2010 and Yadav et al., 2011). This paper has been organized as follows:

Section 2, briefly introduces state about the description of the discrete -time plant with state delay and its transformation, section 3 , describes the method for calculating the gain matrix, section 4, illustrates proposed design of nonlinear sliding surface, section 5, illustrates the control law by proposed algorithm. Section 6, reveals the experimental simulated results analysis and section 7 , and describes the conclusion.

\section{Problem Statement and Description}

Consider the following nonlinear discrete time system:

$$
\begin{aligned}
& x(k+1)=f(x(k), u(k), k), \\
& x(k+1)=(A+\delta A) x(k)+B u(k) \\
& x(k+1)=(A+\delta A) x(k)+B u(k) \\
& y(k)=C x(k)
\end{aligned}
$$

where $x(k) \in \mathfrak{R}^{n}$, are states vector, $u(k) \in \mathfrak{R}^{m}$ is control input vector, $y(k) \in \mathfrak{R}^{p}$ is the output respectively and $f(k)$ is the nonlinear function. The term $A, B$ and $C$ are some constant matrices of appropriate dimensions and parameters and $k$ is the sampling time. We assume the following assumptions:

2.1 Assumption $I$ : the pair $(A, B)$ is controllable and the pair $(A, C)$ is observable, matrices $B$ and $C$ have full rank.

2.2 Assumption II : $p>m$ and $\operatorname{rank}(A, B)=m$

For analysing the system (1), we will transform it in to its regular form. Lets suppose $B=\left[\begin{array}{ll}B_{1} & B_{2}\end{array}\right]^{T}$ and satisfies the $\operatorname{det}(B) \neq 0$, there exists a linear non-singular transformation

$$
z(k)=M x(k)=\left[\begin{array}{cc}
I_{n x n} & -B_{1} B_{2}^{-1} \\
0 & B_{2}^{-1}
\end{array}\right] x(k)
$$

transformation of the system dynamics (1) into its regular form

$$
\begin{aligned}
& z(k+1)=(F+\delta F) z(k)+G(u(k)+f(k)) \\
& y(k)=H z(k)
\end{aligned}
$$

transformed system can be shown without loss of generality.

$$
\begin{gathered}
z_{1}(k+1)=\left(F_{11}+\delta F_{11}\right) z_{1}(k)+\left(F_{12}+\delta F_{12}\right) z_{2}(k) \\
z_{2}(k+1)=\left(F_{21}+\partial F_{21}\right) z_{1}(k)+\left(F_{22}+\partial F_{22}\right) z_{2}(k)+I_{m}\left(\left(I+\partial G_{2}\right) u(k)+\partial f(k)\right) \\
y(k)=H z(k)
\end{gathered}
$$

where $z_{1}(k) \in \mathfrak{R}^{(n-m)}$ and $z_{2}(k) \in \mathfrak{R}^{m}$, are the state vectors and the matrices $F, G_{2}$ are the constant, with their appropriate dimensions and $f(k)$ is the input uncertainties. The following matrix can be written as follows, after transformation. Regular form of the matrix equation can be written as follows:

$$
\begin{gathered}
F=M A M^{-1} \\
F=\left[\begin{array}{ll}
F_{11} & F_{12} \\
F_{21} & F_{22}
\end{array}\right] \\
F_{\text {reg }}=\left[\begin{array}{ll}
F_{11} & F_{12} \\
F_{21} & F_{22}
\end{array}\right]
\end{gathered}
$$

regular form of delayed state of the system is as follows: 


$$
\begin{array}{r}
\delta F_{\text {reg }}=\left[\begin{array}{ll}
\delta F_{11} & \delta F_{12} \\
\delta F_{21} & \delta F_{22}
\end{array}\right] \\
G=M B\left[\begin{array}{c}
0 \\
I_{m}
\end{array}\right] \\
H=C M^{-1}
\end{array}
$$

$z_{1}(k) \in \mathfrak{R}^{(n-m)}$ and $z_{2}(k) \in \mathfrak{R}^{m}$ are the state vectors. $F_{11}, F_{12}, F_{21}, F_{22}$, are known constant matrices with appropriate dimensions. $I_{m}$ is $m \times m$ identity matrix.

\section{Calculation of Optimal Gain Matrix}

From the discrete-time delay system, the gain matrix can be calculated as follows:

$$
x(k+1)=(A+\delta A)+(B+\delta f) u(k)
$$

minimized the cost function

$$
J=\frac{1}{2} \sum_{0}^{\infty} x^{T}(k) Q x(k)+u^{T}(k) R u(k)
$$

to find out the gain with the help of LQR method and the resulting optimal gain matrix is as follows

$$
\begin{gathered}
Q=\operatorname{diag}\left(Q_{1}, Q_{2}, Q_{3}\right) \\
K=\operatorname{dlqr}(A, B, Q, R)
\end{gathered}
$$

\section{Designing of Nonlinear Sliding Surface}

In this section the designing of the nonlinear sliding surfaces for the general MIMO systems. Without loss of generality, the system described by (1) can be transformed in to regular form as follows

$$
\begin{gathered}
z_{1}(k+1)=F_{11 e q} z_{1}(k)+F_{12 e q} z_{2}(k) \\
z_{2}(k+1)=F_{21 e q} z_{1}(k)+F_{22 e q} z_{2}(k)
\end{gathered}
$$

where $F_{11 e q}=F_{11}+\delta F_{11}, F_{12 e q}=F_{12}+\delta F_{12}, F_{21 e q}=F_{21}+\delta F_{21}$, and $F_{22 e q}=F_{22}+\delta F_{22}, G_{2 e q}=G_{2}+\delta G_{2}$

$$
z(k)=M x(k)\left[\begin{array}{l}
z_{1}(k) \\
z_{2}(k)
\end{array}\right]
$$

proposed sliding surface of the system in regular form is as follows

$$
\sigma(k)=\mu^{T} z(k)=0
$$

without loss of generality of the system, sliding condition can be represented as follows:

$$
\begin{aligned}
\sigma(k) & =\mu^{T} z(k) \\
& =\left[\begin{array}{ll}
\mu_{1} & I_{m}
\end{array}\right]\left[\begin{array}{l}
z_{1}(k) \\
z_{2}(k)
\end{array}\right]
\end{aligned}
$$

where $\mu^{T} \in \mathfrak{R}^{m \times n}, \mu_{1} \in \mathfrak{R}^{m(n-m)}$ and $I_{m} \in \mathfrak{R}^{m \times n}$ dimensions. Condition for sliding motion is as follows:

$$
\begin{aligned}
\sigma(k)= & \mu^{T} z(k)=0 \\
= & {\left[\begin{array}{ll}
\mu_{1} & I_{m}
\end{array}\right]\left[\begin{array}{c}
z_{1}(k) \\
z_{2}(k)
\end{array}\right]=0 } \\
& \mu_{1} z_{1}(k)+z_{2}(k)=0 \\
& z_{2}(k)=-\mu_{1} z_{1}(k)
\end{aligned}
$$

seeing $\quad z_{2}(k)$ as dummy input of subsystem (8)

$$
z_{1}(k+1)=F_{11 e q} z_{1}(k)+F_{12 e q} z_{2}(k)
$$




$$
\begin{gathered}
z_{2}(k+1)=F_{21 e q} z_{1}(k)+F_{22 e q} z_{2}(k) \\
\delta F_{11}=D_{1} \Pi(k) E_{1} \\
\delta F_{12}=D_{1} \Pi(k) E_{2}
\end{gathered}
$$

where $\Pi(k) \prod^{T}(k) \leq I, D_{1}, E_{1}, E_{2}$ are known real matrices of appropriate dimensions and $\Pi(k)$ is unknown matrix function with Lebesque-measureable elements and satisfy $\Pi(k) \Pi^{T}(k) \leq I$ and $I$ is the Identity matrix with $m \times m$ dimensions.

$$
\begin{aligned}
& \sigma(k)=\left[K-\rho(y(k))\left\{F_{12 e q}^{T} P\left(F_{11 e q}-F_{12 e q} * K\right)\right\} \quad I_{m}\right]\left[\begin{array}{c}
z_{1}(k) \\
z_{2}(k)
\end{array}\right] \\
& \mu_{1}=\left[K-\rho(y(k))\left\{F_{12 e q}^{T} P\left(F_{11 e q}-F_{12 e q} K\right)\right\}\right] \\
& z_{1}(k+1)=F_{11 e q} z_{1}(k)+F_{12 e q} \rho(y(k)) F_{12 e q}^{T} P F_{11 e q} z_{1}(k)
\end{aligned}
$$

seeing $z_{2}(k)$ as dummy input of the subsystem (9). Substituting (12) into subsystem (9) yields

$$
\begin{aligned}
& z_{1}(k+1)=F_{11 e q} z_{1}(k)-F_{12 e q} \mu_{1} z_{1}(k) \\
& z_{1}(k+1)=\left(F_{11 e q}-F_{12 e q} \mu_{1}\right) z_{1}(k)
\end{aligned}
$$

nonlinear sliding surface can be designed by incorporating the nonlinearity $\rho(y(k))$ in the system dynamics for proven for its stability. If the nonlinear surface is stable then the all the states trajectory will be on the stable surface for future all the time. If the nonlinear surface is stable then the trajectory will be stable on the surface for future all time. The condition for the stability of the nonlinear surface as follows:

$$
2 \rho(y(k))+\rho(y(k)) F_{12 e q}^{T} P F_{12 e q} \rho(y(k)) \leq 0
$$

4.1 Proof: The proof for the nonlinear sliding surface is as follows:

Consider Lyapunov functional candidate for proving the nonlinear sliding surface as stable

$$
\begin{aligned}
V_{1}(k) & =z_{1}^{T}(k) P z_{1}(k) \\
\partial V(k) & =V(k+1)-V(k) \\
\partial V_{1}(k)= & z_{1}^{T}(k+1) P z_{1}(k+1)-z_{1}^{T}(k) P z_{1}(k) \\
z_{1}(k+1)= & F_{11} z_{1}(k)+F_{12}\left(-\mu_{1} z_{1}(k)\right) \\
& \left.=\left(F_{11}-F_{12} \mu_{1}\right) z_{1}(k)\right) \\
& \left.=F_{11 e q}-F_{12 e q}(K-\rho(y(k))) F_{12 e q}^{T} P\left(F_{11 e q}-F_{12 e q} K\right)\right) z_{1}(k) \\
& \left.=F_{11 e q} z_{1}(k)+F_{12 e q} \rho(y(k)) F_{12 e q}^{T} P F_{11 e q} z_{1}(k)\right)
\end{aligned}
$$

where $F_{11 e q}=\left(F_{11}-F_{12} K\right)$ and transform state dynamics becomes as follows

$$
\begin{aligned}
& z_{1}(k+1)=F_{11 e q} z_{1}(k)+F_{12} \psi(y(k)) F_{12}^{T} P F_{11 e q} z_{1}(k) \\
& \sigma(k)=\left[K-\rho(y(k))\left\{F_{12 e q}^{T} P\left(F_{11 e q}-F_{12 e q} K\right)\right\} \quad I_{m}\right]\left[z_{1}(k) \quad z_{2}(k)\right]^{T} \\
& \mu_{1}=K-\rho(y(k))\left\{F_{12 e q}^{T} P\left(F_{11 e q}-F_{12 e q} K\right)\right\} \\
& \left.z_{1}(k+1)=F_{1 e q} z_{1}(k)+F_{12 e q} \rho(y(k))\left\{F_{12 e q}^{T} P F_{1 e q}\right)\right\} z_{1}(k)
\end{aligned}
$$

for stability condition of a system $F_{1 e q}=\left(F_{11 e q}-F_{12 e q} K\right)$ must have all the eigenvalues inside the unit disc. Now, we have to prove that the nonlinear sliding surface is stable by the Lyapunov method for the present state. Therefore all the states will be tracking on stable nonlinear sliding surface for all the future time.

4.2 Lemma 1 [Lee et al., 2001]: Given constant matrices $D, E$ and $Y$ a symmetric constant matrices of appropriate dimensions, the following inequality holds:

$$
Y+D E \Pi+E^{T} \Pi^{T} D^{T} \leq 0
$$

where $\Pi(k)$ satisfies $\Pi(k) \Pi^{T}(k) \leq N$, if and only if for some $\lambda>0$. 


$$
Y+\left[\begin{array}{ll}
\lambda^{-1} E^{T} & \lambda D
\end{array}\right]\left[\begin{array}{cc}
N & 0 \\
0 & I
\end{array}\right]\left[\begin{array}{l}
\lambda^{-1} E \\
\lambda D^{T}
\end{array}\right]<0 \text { holds. }
$$

4.3 Theorem 1: If there exists a symmetric and positive definite matrix $P$, some other matrix $M$ and some scalar function $\zeta$ such that reduced order discrete-time system is asymptotically stable via sliding mode surface (13):

$$
\left[\begin{array}{cccc}
-L & * & * & * \\
F_{11} L+F_{12} M & -L & * & * \\
E_{1} L+E_{2} M & 0 & * & * \\
0 & \zeta & 0 & -\zeta I
\end{array}\right]
$$

where $-L=P^{-1}, M=\mu_{1} P^{-1}$ and $*$ denotes the transposed elements in the symmetric positions.

4.5 Proof : Consider the Lyapunov function candidate for discrete-time system

$$
V(k)=z_{1}^{T}(k) P z_{1}(k)
$$

where $P$ and $Q$ are positive definite symmetrical matrices. The change in Lyapunov function $V(k)$ is

$$
\begin{aligned}
& \partial V(k)=V(k+1)-V(k) \\
& \partial V(k)=z_{1}^{T}(k+1) P z_{1}(k+1)-z_{1}^{T}(k) P z_{1}(k) \\
& \partial V(k)=\left[F_{11 e q}^{T} z_{1}(k)\right]^{T} \times P \times\left[F_{11 e q}^{T} z_{1}(k)\right] \\
& =\Psi^{T}(k)\left[\begin{array}{ll}
F_{11 e q}^{T} & \partial F_{11 e q}^{T}
\end{array}\right]^{T} P\left[\begin{array}{ll}
F_{11 e q}^{T} & \partial F_{11 e q}^{T}
\end{array}\right] \Psi(k)-\Psi^{T}(k)\left[\begin{array}{cc}
P-Q & 0 \\
0 & Q
\end{array}\right] \Psi(k) \\
& \Psi^{T}(k)\left\{\left[\begin{array}{ll}
F_{11 e q} & \partial F_{11 e q}
\end{array}\right]^{T} P\left[\begin{array}{ll}
F_{11 e q} & \partial F_{11 e q}
\end{array}\right]+S\right\} \Psi(k)<0
\end{aligned}
$$

where $\Psi(k)=\left[\begin{array}{c}z_{1}^{T}(k) \\ z_{2}^{T}(k)\end{array}\right], S=\left[\begin{array}{cc}Q-P & 0 \\ 0 & -Q\end{array}\right], F_{1}=\left(F_{11 e q}-F_{12 e q} K\right)$ and $\partial F_{1}=\left(\partial F_{11 e q}-\partial F_{12 e q} K\right)$

Therefore sliding mode dynamics (13) is asymptotically stable, if the following inequality holds

$$
\left\{\left[\begin{array}{ll}
F_{11 \text { eq }} & \partial F_{11 e q}
\end{array}\right]^{T} P\left[\begin{array}{ll}
F_{11 \text { eq }} & \partial F_{11 e q}
\end{array}\right]+S\right\}<0
$$

Apply Schur complements in (14) is equal to

$$
\left[\begin{array}{ccc}
Q-P & * & * \\
0 & -Q & * \\
F_{11} & \partial F_{11} & -P^{-1}
\end{array}\right]<0 \text { condition hold. }
$$

* denotes the transposed elements in the symmetric position. It will be negative definite and the change in Lyapunov function will be negative definite $\partial V(k)<0, \quad z_{1}(k) \neq 0, \quad k=1,2, \ldots .$. and the system will be asymptotically stable.

\section{Designing of Control Law}

The designing of the control law is based upon the stable nonlinear sliding mode control which already proven for its stability.

The sliding mode condition is as follows:

$$
\begin{gathered}
\sigma(k)=\mu^{T} z(k) \\
\sigma(k+1)=\mu^{T} z(k+1)
\end{gathered}
$$

$$
\begin{gathered}
\sigma(k+1)=\sigma(k)=0 \\
\sigma(k+1)=\mu^{T} z(k+1) \\
\mu^{T} z(k+1)=\mu^{T}[(F+\partial F) z(k)+G u(k)]=0 \\
\mu^{T}[(F+\partial F) z(k)+G u(k)]=0 \\
u(k)=\left(-G \mu^{T}\right)^{-1} * \mu^{T}[(F+\partial F) z(k)
\end{gathered}
$$




$$
u(k)=\left(-\mu^{T} G\right)^{-1} *\left[\mu^{T}(F+\partial F) z(k)\right]
$$

At the sampling instant of discrete-time system, the state trajectory will be reaching onto the hyper plane and remain on the sliding surface for due course of time. It is proven, that the control law (19) drives the system trajectory onto the nonlinear sliding surface and maintains the trajectory on the sliding surface during subsequent time. This algorithm will be more effective for highly unstable systems.

\section{Experimental simulated results}

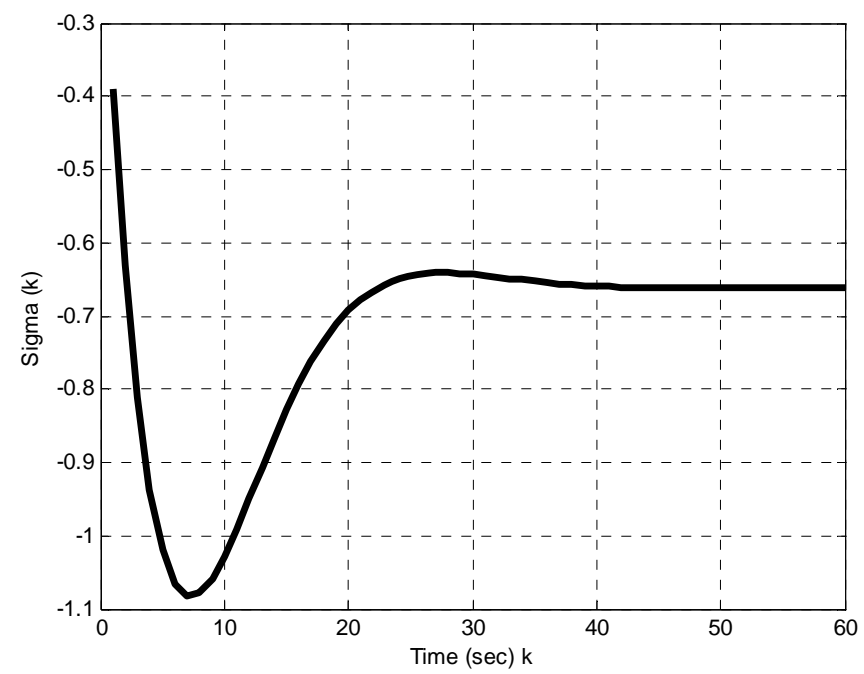

Figure 1. Stable sliding surface

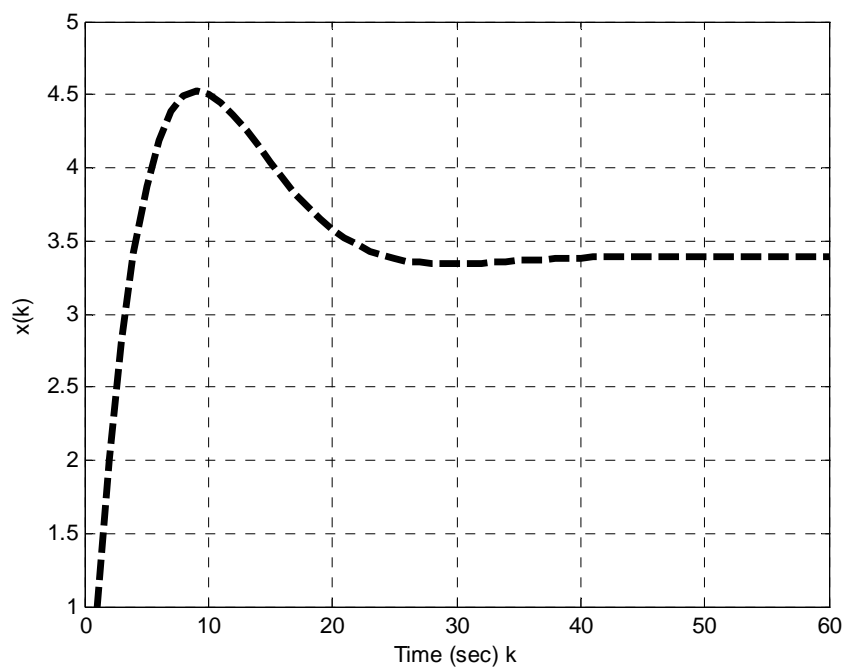

Figure 2. Stable state of the system 


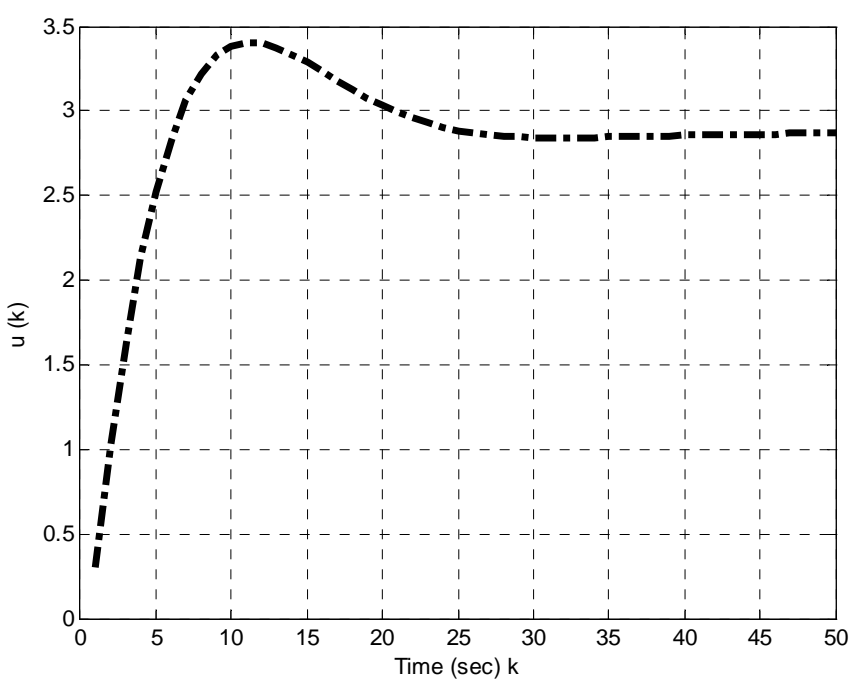

\section{Conclusion}

Figure 3. Control efforts is stable by proposed technique

In this paper, the stability analysis has been investigated for the nonlinear discrete-time system. The nonlinear discrete-time system has been incorporated with uncertainties and the robust sliding mode controller has been designed for discrete-time system with plant uncertainties. The nonlinear systems are highly unstable and the proposed method more effective for avionic unstable systems and the proposed algorithm will have more effectiveness for solving the nonlinear systems. The application of this proposed technique can be well suited to magnetic tape-drive servo motors to control the current and torque.

\section{References}

Bandhyopadhyay B., Deepak F., "High performance tracking controller for discrete plant using nonlinear sliding surface” IEEE transactions on Industrial Electronics, Vol.56, No.9, 2009.

Bartoszewicz. A,, "Discrete sliding mode control strategies”, IEEE Transactions Industrial Electronics, 1998,Vol. 45, pp. 633-637.

Edward C. and Spurgeon S.K. "Sliding Mode Control," Theory and Application London, U.K.: Taylor \& Francis, 1998.

Fiagbedzi Y. A. and Pearson A. E., "Feedback stabilization of linear autonomous time lag systems", IEEE Transactions on Automatic Control, Vol. 31, pp. 847-855, 1986.

Furuta K., "Sliding mode control of a discrete systems” Systems and Control Letters Vol. 14, no. 2, pp.145-152,1990.

Furukawa Y. and Shimemura E., "Predictive control for systems with time-delay", International Journal of Control, Vol. 37, pp. 399-412, 1983.

Gao W., Wang Y. and Homaifa A., "Discrete-time variable structure control systems," IEEE Trans. Ind. Electronics, Vol. 42, no.2, pp.117-122, Apr, 1995.

Gao W.. 'The theory and design of variable structure control' Beijing science publishing company, 1998(in Chinese)

Ghezawi O.M.E.El, .Zinober A.S.I and S.A.Billings, "Analysis and design of variable structure systems using a geometric," International Journal of Control, Vol.38, No.3, pp. 657-671, Sep, 1983.

Golo G. and Milosavljevic C., "Robust discrete-time chattering free sliding mode control,” Syst. Control Lett., vol. 41, no. 1, pp. 19-28, Sep. 2000.

Hung J.Y, Gao W. and Hung J.C., "Variable structure control: A survey” IEEE Transactions Industrial Electronics, Vol.40, No.1, pp.2-22, Feb, 1993.

Hu Q., Du C., Xie L., Wang Y, “Discrete-time sliding mode control with time-varying surface for hard disk drives”, IEEE Trans. Control System. Technology, 2009, 17, (1), pp. 175-183

Jafarov E. M., "Designing of sliding mode control for multi-inputs systems with multiple states delays", Proc. of the America Control Conference, Vol.2, pp.1139-1143, 2000.

Janardhanan S and Bandhyopadhyay B., "Output feedback discrete-time sliding mode control for time delay systems", IEEE Proc. Control Theory Application, Vol. 153, No. 4, July, 2006.

Lan W., Thum C. K. and Chen B. M., "A hard disk-drive servo system design using composite nonlinear feedback control with optimal nonlinear gain tuning methods”, IEEE Trans. on Ind. Electronics Vol. 57, no. 5, pp. 1735-1744, May, 2010.

Lee H.J., Bae Park Jin and Chen Guanrong, "Robust fuzzy control of nonlinear systems with parametric uncertainties”, IEEE Trans. Fuzzy system Apr,2001, pp 369-379 
Milosavljevic D., "General condition for the existence of a quasi-sliding mode on the switching hyperplane in discrete variable structure systems ”, Automation and remote control Vol. 46, pp. 307-314, year, 1985.

Sarpturk. S.Z., Istefanopulos. Y., and Kaynak. O., "On the stability of discrete-time sliding mode systems”, IEEE Transaction Automatic Control, 1987, AC-32, pp.930-932.

Slotine J. J. E. and Sastry S. S., "Tracking controller of nonlinear systems using sliding surfaces with applications to robot manipulators”, International Journal of Control, Vol. 38 no. 2, pp. 465-392, year, 1983.

Spurgeon S.K., "Hyper-plane design technology for discrete time variable structure control systems ," Int. J. Cont., Vol. 55,no.2, pp. 445-456, Feb, 1992.

Utkin V. I., "Equations of sliding mode in discontinuous systems”, Automation and remote control Vol. 1, no. 2, year, 1972.

Utkin V.I., 'Variable structure control systems with sliding mode' IEEE Trans. On Automat. Control Vol.22, 1977, pp. $212-222$.

Won M., Hedrick J.K., "Discrete time adaptive sliding control of nonlinear systems without matching condition” American control conference Sealtle, Washington, pp. 1309-1313, 1995.

Yadav N. K. and Singh R. K. "Discrete-time nonlinear sliding mode controller”, Int. J. Engg. Sc. \& Tech., Vol. 3, No. 3, 2011, pp. 94-100.

Young K.D., Utkin V.I. and Ozgunar U, “A control engineer's guide to sliding mode control,” IEEE Trans. Control Syst. Technology, Vol. 7, No.3, pp. 328-342, May,1999.

\section{Biographical notes}

N. K. Yadav (S’09) was born in Etawah, Uttar Pradesh, India, on April 02, 1969. He received the AMIE degree in Electronics and Telecommunication Engineering from Institute of Engineers (India), Calcutta, India in 1992, MBA (Marketing \& Personnel) from University of Pune, India 1997 \& 1999 respectively, M. Tech degree in Electrical Engineering with specialization in Control and Instrumentation (Cont. \& Inst.) from Motilal Nehru National Institute of Technology (MNNIT), (Deemed University), Allahabad (UP), in 2003. He is currently working towards the Ph.D. degree, from MNNIT, Allahabad (UP), and India. He is also an Assistant Professor with Electrical and Electronics Engineering Department, Lingaya's University, Faridabad, Haryana, India and a member of executive committee of Institute of Engineers (India) Faridabad Local centre, New Delhi India. His current research interests are in areas of variable structure control, nonlinear system dynamics and control, and robust control techniques.

R. K Singh, senior member IEEE and fellow Institution of Engineers (India), is working as a professor, department of Electrical Engineering, MNNIT, Allahabad. His area of interest is dc-dc converters, electrical vehicle and distributed generation application. He is reviewer of national and international journals, /conferences and working on research projects of government of India. Presently he is chairing IEEE local centre at MNNIT, Allahabad. He is also a student's counsellor of IET local chapter of MNNIT.

Received January 2012

Accepted February 2012

Final acceptance in revised form March 2012 